\title{
Análisis bibliométrico de los artículos publicados en la revista Contabilidad y Negocios en el periodo 2006-2019
}

\section{Oscar Alfredo Díaz-Becerra, Fabricia Silva Da Rosa, Mariana Gisella Montes Bravo}

Departamento Académico de Ciencias Contables Pontificia Universidad Católica del Perú

Departamento de Ciências Contábeis Universidade Federal de santa Catarina

Departamento Académico de Educación Pontificia Universidad Católica del Perú

Es importante conocer el avance y nivel de la producción científica de una disciplina, por lo que la revisión de los artículos publicados en revistas científicas constituye una fuente relevante para el análisis. En ese sentido, en este artículo, se realizó una investigación respecto de los trabajos publicados en la revista Contabilidad y Negocios de la Pontificia Universidad Católica del Perú. Se llevó a cabo una investigación bibliométrica sobre el período 2006-2019 con un diseño descriptivo, longitudinal y retrospectivo. A través del sitio web de la revista, se revisaron los veintiocho números publicados en catorce volúmenes en el periodo señalado mediante el análisis de las categorías definidas en la metodología. Con respecto a los resultados principales, se observó que los temas de mayor preferencia por los autores se ubican en las áreas de administración, contabilidad financiera, finanzas y contabilidad de gestión. Asimismo, se observa que la mayor parte de los artículos son de tipo descriptivo. La mayoría de ellos corresponden a autores con filiación distinta a la institución que edita la revista, con una alta participación de autores de género masculino.

Palabras clave: bibliometría, publicaciones, contabilidad, negocios

\section{Análise bibliométrica dos artigos publicados na revista Contabilidad y Negocios no período 2006-2019}

É importante conhecer o progresso e o nível de produção científica de uma disciplina para que a revisão de artigos publicados em revistas científicas se constitua como uma fonte relevante dessa análise, portanto, neste artigo, foram realizadas pesquisas sobre os trabalhos publicados na Revista de Contabilidade e Negócios da Pontifícia Universidade Católica do Peru no período de 2006 a 2019, por meio de uma pesquisa bibliométrica. A pesquisa possui um desenho descritivo, longitudinal e retrospectivo, aplicado às 28 ediçóes publicadas em 14 volumes, por meio da análise das categorias definidas na metodologia, as quais foram acessadas no site da revista. Como principais resultados, observou-se que os temas de maior preferência pelos autores estão localizados nas áreas de administraçáo, Contabilidade Financeira, Finanças e Contabilidade Gerencial. Da mesma forma, observa-se que a maioria dos artigos é descritiva e a maioria corresponde a autores com afiliação diferente à instituição que publica a revista, com alta participação de autores do sexo masculino.

Palavras-chave: bibliometria, publicações, contabilidade, negócios 


\section{Bibliometric analysis of the articles published in the journal Contabilidady Negocios in the period 2006-2019}

It is important to take knowledge of the progress and level of scientific production of a discipline, so the review of articles published in scientific journals constitutes a relevant source for this analysis. Thus, in this article, research was conducted regarding of the works published in the Accounting and Business Magazine of the Pontificia Universidad Católica del Perú in the period 2006 to 2019, through bibliometric research. The research has a descriptive, longitudinal and retrospective design, applied to the 28 issues published in 14 volumes, through the analysis of the categories defined in the methodology, which were accessed from the journal's website. As main results, it was observed that the topics of greatest preference for the authors are located in the areas of administration, Financial Accounting, Finance and Management Accounting. Likewise, it is observed that most of the articles are descriptive and most of them correspond to authors with a different affiliation to the institution that publishes the magazine, with a high participation of male authors.

Keywords: bibliometry, publications, accounting, business

\section{Introducción}

La producción científica sobre contabilidad y administración supone un ciclo que implica la generación de ideas, el desarrollo de la investigación y la comunicación de la misma (Brunozi, Emmerdoerfer, Abrantes \& Klein, 2011). De hecho, la misma producción científica $y$, sobre todo, la productividad científica han sido foco de varias discusiones (Imasato, Perlim \& Borenstein, 2017), ya que el análisis de estos temas contribuye a la difusión de la ciencia; además, aporta contribuciones teóricas, prácticas y metodológicas a los diversos temas cubiertos en estas áreas del conocimiento (Lunkes, Ripoll-Feliu \& Silva da Rosa, 2013). Ya que existe un número limitado de revistas científicas sobre contabilidad, es importante identificar y analizar los temas estudiados y la coherencia de los métodos de investigación aplicados (Lunkes, et al. 2013).

Uno de los medios utilizados para verificar y comprender el avance en las publicaciones académicas consiste en estudiar las publicaciones en las principales revistas (Kremer, Luz, Suave \& Lunkes, 2014). En ese sentido, los estudios bibliométricos son importantes para acompańar las publicaciones, ya que favorecen tanto la evaluación de la producción científica, como el monitoreo de temas y áreas de investigación científica. Siguiendo esa línea, los estudios bibliométricos en el área de contabilidad y negocios han contribuido a la formación de académicos y profesionales al revelar las contribuciones teóricas, prácticas y metodológicas de los artículos publicados en las áreas de contabilidad y negocios; esto se debe a que el tipo de estudios mencionado da información pertinente sobre autores, instituciones, revistas y países que desarrollan temas específicos, lo que supone un aporte para el aprendizaje y la enseñanza en el ámbito académico (Brunozi et al., 2011; Lunkes et al., 2013; Pereira, Souza \& Lunkes, 2013; Ribeiro, 2013; Ribeiro \& Costa, 2013; Kremer et al., 2014; Reina, Ensslin, Ensslin \& Reina, 2016; Carvalho, Pierri, Lunkes \& Gasparetto, 2016; Imasato et al., 2017; Smolski, Dalcin, Visentini \& Bamberg, 2017).

Además de la relevancia de la producción científica para generar conocimiento, también se debe considerar que la misma disciplina y, especialmente, los investigadores en diferentes partes del mundo han sido presionados para publicar: se trata de un medio 
para demostrar su desempeño, pero alerta también la preocupación por el productivismo. Según Imasato et al. (2017), el productivismo se ha debatido en diferentes espacios académicos, como en Estados Unidos, Reino Unido, Australia y Brasil. En esos casos, se ha revelado que la producción científica es un elemento fundamental para la evaluación de proyectos de investigación, calidad de los programas de posgrado o para la evaluación del desempeño académico. Más allá de esta preocupación, diversos autores coinciden en que los estudios respecto de las publicaciones científicas son oportunos y necesarios tanto para evaluar la producción como para comprender la evolución de la ciencia, apreciación aplicable al área de contabilidad y gestión (Bordons \& Zulueta, 1999; Pérez \& Lutsak-Yaroslava, 2017; Sánchez-Perdomo, Rosario-Sierra, Herrera-Vallejera, Rodríguez-Sánchez \& Carrillo-Calvet, 2018; Giménez, 2018; Duque \& Cervantes-Cervantes, 2019). Por ello, en esta investigación se busca analizar las publicaciones realizadas en la revista de Contabilidad y Negocios (2020) de la Pontificia Universidad Católica del Perú (en adelante, PUCP) con el fin de comprender el perfil de las publicaciones, y revelar sus contribuciones a las áreas de contabilidad y negocios en general.

La revista Contabilidad y Negocios fue fundada en el año 2006 como una iniciativa de los docentes del Departamento Académico de Ciencias Contables de la PUCP. La revista se publica una vez al semestre, y presenta artículos de las disciplinas de contabilidad, administración, economía y áreas afines. Contabilidad y Negocios se encuentra indexada en bases de datos de reconocimiento internacional, como Dialnet, BASE, CLASE, DOAJ, EBSCO Host, Journal TOCs, Latindex, ProQuest, Redalyc, REDIB, WorldCat, Primo Central, QUALIS (Clasificación B1) y MIAR (ICDS 9.6).
En esta investigación, se presentan los autores y lectores de la revista a partir de su perfil y evolución. Considerando que, hasta la fecha de culminación de esta investigación, la revista ha publicado veintiocho números en catorce volúmenes, se torna relevante conocer, entre otros aspectos, las áreas de conocimiento correspondientes a los artículos durante todo el periodo de análisis establecido. En este sentido, se ha establecido como objetivo de esta publicación analizar la producción científica de los artículos publicados en la revista Contabilidad y Negocios en el periodo 2006-2019.

La revista Contabilidad y Negocios tiene como finalidad promover y difundir la producción de artículos científicos en las carreras de contabilidad, administración, economía y áreas afines. En tal sentido, cobra relevancia tomar conocimiento sobre la producción científica publicada en la revista desde la fecha de su fundación en el año 2006 hasta el año 2019, de acuerdo a las categorías de análisis descritas en la sección sobre la metodología. Los resultados y el análisis correspondiente permitirán contar con información relevante sobre las tendencias de investigación desarrolladas por los autores que han colaborado con la publicación de sus trabajos en la revista. Así, en este artículo, se muestra que la revista Contabilidady Negocios publica investigaciones sobre temas emergentes y actuales sobre contabilidad y gestión, con énfasis en el contexto de Latinoamérica y los cambios de la contabilidad.

\section{Revisión de la literatura}

Con el tiempo, la forma de gestionar las organizaciones ha evolucionado e incorporado nuevos conceptos y herramientas de toma de decisiones (Spraakman \& Jackling, 2014). Esta realidad llevó a una mayor demanda de profesionales con amplios conocimientos 
para apoyar la toma de decisiones, la gestión, y la formulación e implementación de la estrategia comercial (Jiménez, Gasparetto, Lunkes \& Schnorrenberger, 2017). Esto se debe a que las organizaciones necesitan más conocimiento sobre los factores que afectan sus negocios, como la globalización, los factores culturales e institucionales, y las restricciones de gestión que afectan a todas las estrategias (Krivogorsky \& Ballam, 2019). En respuesta a estas demandas, la comunidad científica reflexiona sobre los estándares de contabilidad y gestión, y los factores organizativos; a partir de ello, establece nuevas formas de ayudar a las organizaciones en la gestión empresarial, y en el cumplimiento de los aspectos formales y legales de la contabilidad. Esta respuesta puede verse de varias maneras, como a) la capacitación de profesionales, b) el desarrollo de métodos, herramientas y estándares normativos, y c) publicaciones científicas.

Las publicaciones se presentan principalmente en revistas evaluadas periódicamente por bases nacionales e internacionales. Esto ha ayudado tanto en la evaluación de la calidad de la producción, como en la difusión del conocimiento científico sobre contabilidad y gestión de las organizaciones (Brunozi et al., 2011; Lunkes et al., 2013; Pereira et al., 2013; Ribeiro, 2013; Ribeiro \& Costa, 2013; Kremer et al., 2014; Reina, et al., 2016; Carvalho et al., 2016; Imasato et al., 2017; Smolski et al., 2017; Rosa \& Romani-Dias, 2019). Específicamente en Brasil (Brunozi et al., 2011) y en América Latina (Lunkes et al., 2013), la investigación contable ha mostrado un crecimiento notable en los últimos años, especialmente con respecto a los factores relacionados a los nuevos programas de posgrado; la oferta de cursos de especialización; y la evolución en los medios de difusión científica, como en reuniones, seminarios, congresos y revistas científicas. En este sentido, como se observa en la tabla 1, los estudios se han basado en la produc- ción científica para analizar el estado y la evolución de las áreas de contabilidad y gestión de negocios (Brunozi et al., 2011; Lunkes et al., 2013; Pereira et al., 2013; Ribeiro, 2013; Ribeiro \& Costa, 2013; Kremer et al., 2014; Reina, et al., 2016; Carvalho et al., 2016; Imasato et al., 2017; Smolski et al., 2017; Rosa \& Romani-Dias, 2019).

Brunozi et al. (2011) llevaron a cabo una investigación bibliométrica en la Revista Contabilidade \& Finanças de la Universidad de San Paulo (USP) de 1989 a 2009. Concluyeron que un hito de la revista fue el año 2001, en el que se comenzó a presentar una periodicidad constante de las publicaciones y una mayor apertura a la adhesión de diferentes autores. Los temas evolucionaron a lo largo de los veinte años de análisis, que se organizan de la siguiente manera: finanzas y contabilidad (29\%), enseńanza de contabilidad y conocimiento (13\%), contabilidad clásica y teoría de la contabilidad (12\%), y sistemas de información, comunicación y divulgación (11\%). Se utilizaron varias fuentes de bibliografía, sobre todo artículos internacionales. En el período analizado, hubo principalmente trabajos descriptivos, principalmente en los primeros ańos de la revista. En cuanto a los medios, se observó una concentración de publicaciones bibliográficas y documentales entre 1989 y 2002. Entre 2003 y 2009, el escenario es diferente, pues los estudios predominantes se realizaron con la entrada de datos primarios y/o secundarios, que pueden clasificarse como empíricos. En el período analizado, hubo una tendencia en la publicación de artículos sobre enseñanza y conocimiento, y teoría contable de manera práctica y empírica a través de modelos en datos primarios y/o secundarios. La explicación de los cambios se debe principalmente a los cambios constantes debido a la estandarización contable global, que requiere interpretaciones del impacto en el patrimonio de las entidades. El conocimiento de 
Tabla 1. Categorías de análisis en la literatura

\begin{tabular}{|c|c|c|c|c|c|c|c|c|c|c|c|}
\hline $\begin{array}{l}\text { Categorías de } \\
\text { análisis }\end{array}$ & $\begin{array}{l}\text { Brunozi } \\
\text { et al., } \\
2011\end{array}$ & $\begin{array}{l}\text { Lunkes } \\
\text { et al., } \\
2013\end{array}$ & $\begin{array}{c}\text { Pereira } \\
\text { et al., } \\
2013\end{array}$ & $\begin{array}{c}\text { Ribeiro, } \\
2013\end{array}$ & $\begin{array}{c}\text { Ribeiro } \\
\text { \& Costa } \\
2013\end{array}$ & $\begin{array}{l}\text { Kremer } \\
\text { et al., } \\
2014\end{array}$ & $\begin{array}{l}\text { Reina, } \\
\text { et al., } \\
2016\end{array}$ & $\begin{array}{l}\text { Carvalho } \\
\text { et al., } \\
2016\end{array}$ & $\begin{array}{l}\text { Imasato } \\
\text { et al., } \\
2017\end{array}$ & \begin{tabular}{|c|} 
Smolski \\
et al., \\
2017
\end{tabular} & $\begin{array}{c}\text { Rosa \& } \\
\text { RomaniDias, } \\
2019\end{array}$ \\
\hline \multicolumn{12}{|c|}{ Afiliación académica } \\
\hline \multicolumn{12}{|l|}{ Análisis de redes } \\
\hline \multicolumn{12}{|l|}{ Año de publicación } \\
\hline \multicolumn{12}{|l|}{ Autor por artículo } \\
\hline \multicolumn{12}{|l|}{ Bases de datos } \\
\hline \multicolumn{12}{|l|}{ Citas } \\
\hline \multicolumn{12}{|l|}{ Fascículos } \\
\hline \multicolumn{12}{|l|}{ Género del autor } \\
\hline \multicolumn{12}{|l|}{ Metodología } \\
\hline \multicolumn{12}{|l|}{ Páginas } \\
\hline \multicolumn{12}{|l|}{ Palabras clave } \\
\hline \multicolumn{12}{|l|}{ Referencias } \\
\hline \multicolumn{12}{|l|}{ Tema } \\
\hline \multicolumn{12}{|l|}{ Título académico } \\
\hline Título de la revista & & & & & & & & & & & \\
\hline
\end{tabular}

los pronunciamientos contables (CPC) y las normas emitidas en las Normas Internacionales de Información Financiera (NIIF) es cada vez más importante. En ese sentido, se observa la importancia que adquirió la revista del estudio, pues se ha convertido principalmente en un medio que permite evaluar y enumerar la investigación sobre contabilidad y finanzas. Por medio de este estudio, es válido enfatizar la importancia de la investigación contable para el crecimiento de los estudios en el área. Además, es necesario observar en los estudios temas relacionados a aspectos metodológicos, temáticos o referenciales, ya que estos aún no se abordan ampliamente en el área de contabilidad académica y pueden contribuir de alguna manera a nuevas investigaciones.
Lunkes et al. (2013) revisaron las publicaciones en revistas brasileñas y españolas, y descubrieron que los resultados ponen de manifiesto que la contabilidad de gestión no ocupa un lugar destacado en las publicaciones en dichas publicaciones. Además, los estudios investigados detectan las oportunidades de mejora en la formación ante la falta de centros especializados en el ámbito de la contabilidad de gestión y en la incorporación de redes consolidadas de autores de diferentes países. Los motivos de esta situación son (i) la ausencia de bases de datos o la dificultad en la obtención de datos, (ii) el número limitado de revistas especializadas en contabilidad de gestión, y (iii) la falta de creatividad de sus investigadores. Los resultados también ayudan a comprender que los investigadores en contabilidad de gestión en Brasil y España tienen 
una fuerte tendencia a la búsqueda de temas periféricos (que han sido incluidos en el presente estudio dentro de la línea de investigación "otros") mediante el estudio de métodos de investigación del caso y revisión, frente a la indagación en temas como costos, y planificación y control. Es interesante destacar el crecimiento y el predominio de los estudios empíricos en Brasil. Se trata de un indicador positivo de la evolución del tipo de método de investigación, ya que los resultados de estos estudios no se basan exclusivamente en una revisión de la literatura.

Pereira et al. (2013) realizaron un análisis bibliométrico de publicaciones sobre contabilidad en revistas internacionales de turismo en el periodo 1992-2012. Los autores revelan que las revistas destacadas fueron International Journal of Hospitality Management and Worldwide y Hospitality and Tourism Themes. Además, el estudio revela que existe una gran diversidad de revistas de turismo que publican sobre contabilidad. Las palabras clave más utilizadas fueron (i) sector hotelero, (ii) servicios y hospitalidad, (iii) hoteles, (iv) gestión de riesgos, (v) gestión de costos, y (vii) controlador. El área de contabilidad asociada con turismo y hospitalidad es estudiado por varias universidades y autores. Los temas relacionados a la contabilidad y el turismo se asocian principalmente a la contabilidad gerencial y la toma de decisiones, la contabilidad y la gestión de costos, y los indicadores de desempeño financieros y no financieros.

Ribeiro y Costa (2013) llevaron a cabo un análisis bibliométrico y de redes sociales para mapear el perfil y la evolución de la producción científica de la Revista Contemporánea de Contabilidad en el período 2004-2012. En este contexto, la revisión de la literatura identificó algunos trabajos bibliométricos y/o de redes sociales ya realizados específicamente en revistas, es decir, que ya han explorado colecciones de revistas específicamente en el área de contabilidad. Se encontró que la mayoría de los artículos publicados tienen una asociación con al menos dos autores, es decir, corresponde a 89,47\% de los 133 artículos. En el contexto general, hubo un gran número de autores (318) en comparación al número de instituciones de educación superior (93) involucrados en la publicación de los 133 artículos estudiados en nueve años de investigación. El alto número de autores y de instituciones de educación superior fue predominante, aspectos que son inherentes a las revistas que se ocupan de la búsqueda de la diversificación, y socialización de opiniones y puntos de vista.

Para Ribeiro y Costa (2013) los temas sobre contabilidad de costos, enseñanza e investigación, contabilidad ambiental, gobierno corporativo, contabilidad internacional, desempeño organizacional, y control y planificación presupuestaria (gubernamental) fueron los que se destacaron en el periodo de nueve años de la investigación, lo que representa 54,89\% del total de 133 artículos analizados. Dichos datos están en línea con las principales palabras clave que se ven en estos artículos, que fueron contabilidad, gestión, costos, evaluación, desempeño, corporativo, gobierno, medioambiente, información y educación. Se enfatiza la importancia de estos temas para el área de contabilidad, ya que se están abordando cada vez más en publicaciones en el área de administración, contabilidad y finanzas; esto se evidencia, principalmente, en la contabilidad ambiental, el gobierno corporativo y la contabilidad internacional.

Ribeiro y Costa (2013) exploraron la producción académica científica de la Revisión de la Administración de Brasil en el periodo de 2004-2012. El artículo se basó en un estudio de redes sociales y bibliométrica (característica de publicación, cantidad de autor por artículo, autores con más producción, referencias 
utilizadas, instituciones de educación superior), y se utilizaron estadísticas descriptivas en 159 artículos identificados. Los principales resultados de este estudio determinaron el predominio del trabajo en asociación y la centralidad de la red de coautoría; asimismo, las palabras más utilizadas fueron gestión, rendimiento y organización. La publicación de los artículos de la revista refleja el universo de la producción académica en el área de la administración. Esto ayuda a desarrollar, difundir y socializar el área a través de artículos científicos publicados en la literatura académica nacional.

Kremer et al. (2014) llevaron a cabo un estudio bibliométrico en revistas de contabilidad brasileñas entre 2001 y 2013. Identificaron que la contabilidad ha tenido un marcado desarrollo en las últimas décadas con la inserción de nuevos temas y métodos de investigación, la expansión del número de revistas, y, sobre todo, estudios en otras áreas de conocimiento. Uno de los medios utilizados para verificar y comprender este avance consiste en las publicaciones en revistas científicas. Los resultados revelan que, del total de artículos publicados en las revistas que componen la muestra, el $6 \%$ se refiere a la contabilidad de gestión; y los otros, principalmente a la contabilidad financiera y la auditoría. Entre los trabajos de contabilidad de gestión, destacan las líneas de investigación que miden y evalúan el desempeño y las múltiples técnicas. Además, se muestra que, entre los métodos de investigación utilizados, la encuesta y el estudio de caso destacan sobre los demás.

Reina et al. (2016) analizaron el perfil de publicaciones científicas internacionales sobre el tema de la selección de proyectos. Aunque no está directamente relacionado con la contabilidad, el estudio es importante para comprender la relevancia de los estudios bibliométricos y bibliográficos de las revistas. A partir del análisis bibliométrico, es posible identificar las revistas, artículos, autores y palabras clave que se destacaron en el portafolio bibliográfico. Sin embargo, los autores señalan limitaciones importantes de este tipo de estudio, tales como (i) bases de datos disponibles, (ii) uso de procesos estructurados para la selección y divulgación de artículos, autores, revistas y palabras clave relevantes, e (iii) identificación del tema alineado con la investigación.

Carvalho et al. (2016) analizaron el perfil de publicaciones sobre el tema de gestión de la contabilidad en revistas internacionales de contabilidad indexadas a la base de datos ISI (Instituto de Información Científica). Con respecto a la metodología, se utilizó una investigación descriptiva con un enfoque cualitativo. Se seleccionaron artículos que contenían en su resumen, título o palabras clave las palabras «contabilidad de gestión», «control de gestión»y «contraloría». El estudio se centró en el periodo 2001-2015. Los estudios se clasificaron en tema y método de investigación, número de autores, género, y duración de los artículos. Los resultados muestran una inserción débil de la contabilidad gerencial en las publicaciones de las revistas analizadas (6,7\%), mientras la investigación de contabilidad de gestión se posiciona como la publicación periódica con el mayor número de trabajos. Asimismo, la mayoría de los artículos tienen dos autores y el género masculino predomina entre ellos La metodología más utilizada fue el estudio de caso y el tema predominante fue el control organizacional.

Imasato et al. (2017) analizaron el perfil de los doctores académicos que operan en el área de administración en Brasil y sus respectivas publicaciones científicas. El trabajo identificó factores vinculados a la capacitación y la experiencia que influyen, en promedio, en la productividad científica de los académicos de administración. Entre ellos, se destacan los siguientes 
aspectos: (a) el apoyo del gobierno (subvención de investigación), que es un factor positivo y significativo en la productividad científica; (b) los estudios de doctorado en el extranjero demostraron ser un factor positivo para la productividad de los artículos publicados en revistas que figuran en el Scientific Journal Ranking (SJR); (c) el efecto del tiempo desde el final del doctorado influye en la productividad a lo largo del tiempo; y (d) el intervalo doctoral no tuvo un efecto significativo en la productividad. Las preguntas de perfil académico, como la edad, el género o la trayectoria profesional, no se pudieron abordar adecuadamente en esta investigación, pero los autores las consideran relevante para futuras investigaciones.

Smolski et al. (2017) plantean una investigación de corte bibliométrico sobre las transformaciones de los trabajos publicados en la Revista de Administração Pública (RAP) para el periodo de 2003 a 2016. El perfil de los 729 artículos analizados muestra que el número de trabajos desarrollados en conjunto está creciendo. Asimismo, se evidencia que el número de autores por artículo ha aumentado, y hay un predominio de autores hombres en las publicaciones. El contenido de los artículos señala que el enfoque se basa en la ciencia administrativa. Sin embargo, con el paso de los años, el tema está perdiendo espacio, lo que evidencia una diversificación de los artículos de la revista en las perspectivas teóricas utilizadas. otro lado, a pesar de ser el territorio principal explotado, los estudios organizacionales han perdido espacio. En cuanto al diseño, los artículos suelen utilizar estudios de caso; y son aplicados y cuantitativos, propuesta que ha ganado espacio en el período estudiado. El hecho de que muchos trabajos se estén desarrollando en coautoría ha llevado a una comprensión de las razones, causas o consecuencias de esta tendencia. Existe poca colaboración entre pares, lo cual es un tema extremadamente importante para el desarrollo de la ciencia y la administración pública.
Rosa y Romani-Dias (2019) analizaron bases de datos brasileñas, y constataron que las revistas brasileñas de administración, contabilidad y turismo en las diferentes bases científicas que calculan el factor de impacto aportan dos contribuciones centrales al campo: la primera relacionada con el análisis de revistas y la segunda relacionada con medidas de base científica. Se observa, según los datos recopilados, que las revistas brasileñas en el área han tenido un ligero aumento en las tasas calculadas por las bases de datos Scopus y Scielo, lo que indica que esas revistas no han podido aumentar razonablemente el impacto internacional de sus publicaciones. Por lo tanto, se argumenta que solo la presencia en las bases de datos no garantiza la difusión internacional de las revistas. También se nota que todavía existen pocas revistas nacionales presentes en Web of Science, la base internacional principal que calcula el factor de impacto: solo hay tres revistas de 314 sobre el campo que se encuentran en la base de datos mencionadas, es decir, aproximadamente el 1\% de la población. Las tres revistas han tenido un crecimiento razonable con respecto a sus factores de impacto en la Web of Science; sin embargo, se evidencia que este aumento se ha debido sustancialmente a las autocitas, y a citas de otras revistas nacionales y de autores brasileños que publican en revistas extranjeras. Esta situación supone reflexionar sobre la internacionalización real del campo.

A partir de la literatura revisada, se revela la importancia de monitorear la producción técnico-metodológica por parte de la comunidad académica. Así como en Brasil, las revistas de los países latinoamericanos han evolucionado con respecto al tratamiento de contabilidad, pero necesitan más inserción internacional. Entre los resultados principales, se observó la consolidación del campo de contabilidad y gestión en las revistas nacionales e internacionales, lo que puede ser un indicador del vasto campo de conocimiento que tienen las revistas latinoamericanas. 


\section{Metodología}

La investigación tiene un diseño descriptivo, longitudinal y retrospectivo. Se aplicó a los veintiocho números publicados en catorce volúmenes durante el periodo comprendido entre 2006 y 2019. Se accedió a los números de la revista por medio de su sitio web. Las categorías de análisis establecidas se encuentran en la tabla 2 .

Tabla 2. Categoría de análisis

\begin{tabular}{|c|l|}
\hline$N^{\circ}$ & \multicolumn{1}{|c|}{ Descripción } \\
\hline 1 & Temática \\
\hline 2 & Idioma \\
\hline 3 & Metodología aplicada \\
\hline 4 & Artículos por número \\
\hline 5 & Autores por artículo \\
\hline 6 & Autores por número \\
\hline 7 & Páginas por artículo \\
\hline 8 & Páginas por número \\
\hline 9 & Referencias bibliográficas por artículo \\
\hline 10 & Referencias bibliográficas por número \\
\hline 11 & Tiempo de aprobación \\
\hline 12 & Nacionalidad de los autores \\
\hline 13 & Autores por género \\
\hline 14 & Universidad o institución de autores \\
\hline
\end{tabular}

Sobre la base de las categorías establecidas, se realizó un análisis de todos los artículos publicados en los veintiocho números disponibles de la revista en el periodo delimitado para esta publicación. Se tuvo como base el objetivo planteado para este trabajo de investigación bibliométrico. Como se indicó en el párrafo previo, se accedió a los datos mediante el sitio web de la revista, en el que se encuentran disponibles todos los números publicados mediante acceso abierto. El acceso se realizó en el mes de enero de 2020, fecha en que estaba disponible el último número de la revista ((14) $28,2019)$. Los datos fueron procesados mediante el uso de una hoja de cálculo, en la cual se tabularon las catorce categorías de análisis para luego ordenar la información y clasificarla según los datos de coincidencia. Finalmente, estos datos son presentados en las tablas que se describen en el apartado de análisis e interpretación de los resultados de la investigación.

\section{Análisis e interpretación de resultados}

Teniendo en cuenta las categorías de análisis descritas en la metodología, en este apartado, se presentan los resultados correspondientes a cada una de ellas. En este sentido, la primera categoría de análisis se refiere a la temática sobre la que se han publicado los artículos. Este aspecto se muestra en la tabla 3. La información muestra que 48 de los 196 artículos publicados $(24,49 \%$ del total) corresponden a la temática de administración. En segundo lugar, se encuentran 33 artículos (16,84\% del total) sobre actualidad contable. En tercer lugar, se encuentran 28 artículos (14,29\% del total) sobre banca y finanzas. La suma de estas tres temáticas asciende a 109 artículos, que equivale a $55,61 \%$ de los artículos revisados.

Los resultados muestran una marcada tendencia en las tres áreas mencionadas en el párrafo precedente. Esto concuerda con los estudios descritos en la revisión de la literatura que respalda este trabajo y, sobre todo, con las temáticas privilegiadas por el objetivo de la revista. Otras temáticas importantes, que incrementan la participación de las publicaciones en contabilidad, corresponden a los artículos de otras áreas de la disciplina. Entre ellas, se encuentran contabilidad de gestión, auditoría y tributación. De cada una de estas áreas, se han publicado más de diez artículos, que, en total, corresponden a 44 artículos, equivalentes a $22,45 \%$. 
Tabla 3. Artículos según temática

\begin{tabular}{|l|c|c|}
\hline \multicolumn{1}{|c|}{ Descripción } & Artículos & $\%$ \\
\hline Administración & 48 & $24,49 \%$ \\
\hline Actualidad contable & 33 & $16,84 \%$ \\
\hline Banca y finanzas & 28 & $14,29 \%$ \\
\hline Contabilidad de gestión & 19 & $9,69 \%$ \\
\hline Auditoría & 13 & $6,63 \%$ \\
\hline Tributación & 12 & $6,12 \%$ \\
\hline Estrategia empresarial & 8 & $4,08 \%$ \\
\hline Economía & 7 & $3,57 \%$ \\
\hline Contabilidad gubernamental & 6 & $3,06 \%$ \\
\hline Ponencias & 4 & $2,04 \%$ \\
\hline Reseńas & 4 & $2,04 \%$ \\
\hline Costos & 3 & $1,53 \%$ \\
\hline Formación profesional & 3 & $1,53 \%$ \\
\hline Comercio exterior & 1 & $0,51 \%$ \\
\hline Derecho & 1 & $0,51 \%$ \\
\hline Emprendimiento & 1 & $0,51 \%$ \\
\hline Estudio de caso & 1 & $0,51 \%$ \\
\hline Ética & 1 & $0,51 \%$ \\
\hline Evaluación de proyectos & 196 & $0,51 \%$ \\
\hline Opinión & & $0,51 \%$ \\
\hline Tecnología & 1 & $0,51 \%$ \\
\hline Total & $100,00 \%$ \\
\hline
\end{tabular}

La segunda categoría de análisis corresponde al idioma en que fueron publicados los artículos. En la tabla 4, se presenta la información de los tres idiomas en que los artículos se han publicado durante el periodo de análisis. El idioma original de la revista es el español, que corresponde a 157 artículos publicados (80,10\% del total). En segundo lugar, se encuentran 33 artículos (16,84\% del total) publicados en portugués. Por último, se encontraron 6 artículos $(3,06 \%$ del total) en inglés. El primer artículo en portugués fue publicado en la edición número 17 , mientras que el primer artículo en inglés fue publicado en la edición número 19. Esto evidencia la inclinación del Comité Editorial por incorporar publicaciones en los otros dos idiomas de interés para la comunidad académica. Ya que las investigaciones presentadas en la sección de revisión de la literatura no consideran el idioma en que fueron publicados los artículos, los resultados de este estudio son relevantes para tener información sobre la cantidad de artículos publicados en cada uno de los idiomas aceptados por la publicación.

\section{Tabla 4. Artículos según idioma}

\begin{tabular}{|l|c|c|}
\hline \multicolumn{1}{|c|}{ Idioma } & Artículos & $\%$ \\
\hline Espańol & 157 & $80,10 \%$ \\
\hline Portugués & 33 & $16,84 \%$ \\
\hline Inglés & 6 & $3,06 \%$ \\
\hline Total & 196 & $100,00 \%$ \\
\hline
\end{tabular}

La información correspondiente a la metodología revelada en cada artículo publicado se presenta en la tabla 5. Se puede observar que la mayoría de artículos han sido de tipo descriptivo, ya que 140 artículos $(71,43 \%$ del total) presentan este tipo de metodología. En segundo lugar, se presentan 16 artículos (8,16\% del total) de tipo analítico. En tercer lugar, 7 artículos $(3,57 \%$ del total) corresponden a revisión bibliográfica. Por último, se encontraron 5 artículos de metodología cuantitativa; y 5, de estudio de caso. En sus inicios, la revista no restringía la publicación de artículos elaborados sobre la base de una metodología específica. Con el pasar de los años, de acuerdo con las exigencias de las bases de datos de revistas indexadas, Contabilidad y Negocios da preferencia a artículos en los que se evidencia un mayor rigor en la metodología científica aplicada en las investigaciones propuestas para su publicación. Los resultados de esta investigación guardan relación con la evolución descrita en las publicaciones analizadas en la revisión de la literatura, 
pues, como en los casos previamente comentados, se observa una situación similar con respecto a la concentración de artículos descriptivos en los primeros años de creación de la revista. Asimismo, esta situación también va cambiando a publicaciones de mayor rigor en la metodología aplicada.

Tabla 5. Artícullos según metodología

\begin{tabular}{|l|c|c|}
\hline \multicolumn{1}{|c|}{ Metodología } & Artículos & $\%$ \\
\hline Descriptiva & 140 & $71,43 \%$ \\
\hline Analítica & 16 & $8,16 \%$ \\
\hline Revisión bibliográfica & 7 & $3,57 \%$ \\
\hline Cuantitativa & 5 & $2,55 \%$ \\
\hline Estudio de caso & 5 & $2,55 \%$ \\
\hline Análisis exploratorio & 4 & $2,04 \%$ \\
\hline Empírica & 4 & $2,04 \%$ \\
\hline Aplicada & 3 & $1,53 \%$ \\
\hline Cualitativa & 2 & $1,02 \%$ \\
\hline Bibliométrica & 1 & $0,51 \%$ \\
\hline Correlativa & 1 & $0,51 \%$ \\
\hline Enfoque comparativo & 1 & $0,51 \%$ \\
\hline Exploratoria & 1 & $0,51 \%$ \\
\hline Método de los expertones & 1 & $0,51 \%$ \\
\hline Metodología de las distancias & 1 & $0,51 \%$ \\
euclidianas & 1 & $0,51 \%$ \\
\hline Modelo de ecuaciones estructurales & 1 & $0,51 \%$ \\
\hline Modelo de regresión & 1 & $0,51 \%$ \\
\hline Modelo econométrico probit & 196 & $100,00 \%$ \\
\hline Regresión múltiple & & \\
\hline Total & 1 & $0,51 \%$ \\
\hline & 1 & \\
\hline
\end{tabular}

En la tabla 6, se presenta la información relacionada a la cantidad de artículos publicados en cada uno de los 28 números. Se puede observar que la cantidad varía desde 5 artículos como mínimo por número hasta un máximo de 9. Los números con 6 y 8 artículos son los que tienen mayor presencia: en cada caso,

corresponden a 9 números $(32,14 \%$ del total en cada caso). En segundo lugar, se encuentran 4 números con 7 artículos (14,29\% del total). Asimismo, el promedio de artículos publicados por cada número es de 7 artículos. A pesar de no contar con información similar en las investigaciones analizadas en la revisión de la literatura que respalda este trabajo, es importante conocer este tipo de información. En los últimos años, el número de artículos se encuentra por encima del número mínimo exigido por algunas bases de datos indexadas.

Tabla 6. Artícullos por número

\begin{tabular}{|c|c|c|}
\hline Cantidad de artículos & Números & $\%$ \\
\hline 5 & 3 & $10,71 \%$ \\
\hline 6 & 9 & $32,14 \%$ \\
\hline 7 & 4 & $14,29 \%$ \\
\hline 8 & 9 & $32,14 \%$ \\
\hline 9 & 3 & $10,71 \%$ \\
\hline Total & 28 & $100,00 \%$ \\
\hline
\end{tabular}

Otra de las categorías de análisis establecida en la metodología corresponde a la cantidad de autores por artículo, información que se presenta en la tabla 7. Se observa que casi la mitad de artículos han sido elaborados por un solo autor. Estos corresponden a 97 artículos (49,49\% del total). En segundo lugar, se ubican 44 artículos $(22,45 \%$ del total) escritos por 3 autores. En tercer lugar, se ubican 32 artículos (18,88\% del total) escritos por 2 autores. Es importante destacar que los artículos con un solo autor tienen mayor presencia en los primeros números. Por otra parte, en los últimos números, aumenta la presencia de artículos con más de un autor, aspecto que es similar a las conclusiones de las investigaciones descritas en la revisión de la literatura; asimismo, se enmarca en la tendencia de privilegiar el trabajo en equipo más que el trabajo individual. 
Tabla 7. Autores por artículo

\begin{tabular}{|c|c|c|}
\hline Cantidad de autores & Artículos & $\%$ \\
\hline 1 & 97 & $49,49 \%$ \\
\hline 2 & 37 & $18,88 \%$ \\
\hline 3 & 44 & $22,45 \%$ \\
\hline 4 & 14 & $7,14 \%$ \\
\hline 5 & 4 & $2,04 \%$ \\
\hline Total & 196 & $100,00 \%$ \\
\hline
\end{tabular}

Tabla 8. Nacionalidad de los autores

\begin{tabular}{|l|c|c|}
\hline \multicolumn{1}{|c|}{ País } & Autores & $\%$ \\
\hline Brasil & 122 & $32,19 \%$ \\
\hline Perú & 90 & $23,75 \%$ \\
\hline Espańa & 76 & $20,05 \%$ \\
\hline Colombia & 22 & $5,80 \%$ \\
\hline Chile & 21 & $5,54 \%$ \\
\hline México & 9 & $2,37 \%$ \\
\hline Ecuador & 8 & $2,11 \%$ \\
\hline Uruguay & 7 & $1,85 \%$ \\
\hline Portugal & 6 & $1,58 \%$ \\
\hline Argentina & 5 & $1,32 \%$ \\
\hline Cuba & 4 & $1,06 \%$ \\
\hline Estados Unidos & 3 & $0,79 \%$ \\
\hline Venezuela & 2 & $0,53 \%$ \\
\hline China & 1 & $0,26 \%$ \\
\hline Costa Rica & 1 & $0,26 \%$ \\
\hline Japón & 1 & $0,26 \%$ \\
\hline Nicaragua & 1 & $0,26 \%$ \\
\hline Total & 379 & $100,00 \%$ \\
\hline
\end{tabular}

Para identificar la nacionalidad de los autores, se cuenta con la información de la tabla 8. En primer lugar, 122 autores $(32,19 \%$ del total) son de nacionalidad brasilera. En segundo lugar, se encuentran 90 autores $(23,75 \%$ del total) de nacionalidad peruana. En tercer lugar, se identifican 76 autores $(20,05 \%$ del total) de nacionalidad española. En contraste a lo evidenciado en los primeros años de la revista, estos resultados muestran el interés de autores de distintas nacionalidades por publicar en Contabilidad $y$ Negocios, en la que no hay preferencias para aceptar artículos de una nacionalidad en particular.

En la tabla 9, se presenta la información relacionada a la categoría de análisis referida a la cantidad de autores incorporados en cada número publicado de la revista. En 11 números $(39,29 \%$ del total), la cantidad de autores se ubica en el rango de 6 a 10 . En segundo lugar, se encuentran 7 números con autores que se ubican en el rango de 11 a 15. En tercer lugar, se encuentran 5 números $(17,86 \%$ del total en cada caso) con rangos de 16 a 20 y 21 a 25 autores. Estos resultados se relacionan con lo explicado en la tabla 7 sobre la cantidad de autores por artículos.

Tabla 9. Autores por número

\begin{tabular}{|c|c|c|}
\hline Cantidad de autores & Números & $\%$ \\
\hline de 06 a 10 & 11 & $39,29 \%$ \\
\hline de 11 a 15 & 7 & $25,00 \%$ \\
\hline de 16 a 20 & 5 & $17,86 \%$ \\
\hline de 21 a 25 & 5 & $17,86 \%$ \\
\hline Total & 28 & $100,00 \%$ \\
\hline
\end{tabular}

La siguiente categoría de análisis corresponde a la cantidad de páginas por artículo publicado, cuyos resultados se presentan en la tabla 10 . Se observa que 59 artículos (31,10\% del total) tienen una extensión que abarca entre 16 y 20 páginas. El segundo lugar es compartido por dos grupos de 49 artículos (25\% del total en cada caso): el primero presenta un rango de 6 a 10 páginas; y el segundo, de 11 a 15 páginas. Asimismo, el promedio de páginas por artículo de los 196 artículos publicados es de 14 páginas. Estos resultados se encuentran alineados a las pautas para 
los autores, tanto a las establecidas para los primeros números publicados por la revista como a las pautas vigentes. El promedio de páginas por artículo publicado permite incluir una extensión adecuada para la presentación de todos los puntos que debe considerar un artículo científico de calidad mínima.

Tabla 10. Páginas por artículo

\begin{tabular}{|c|c|c|}
\hline Páginas por artículo & Artículos & $\%$ \\
\hline de 1 a 5 & 19 & $9,69 \%$ \\
\hline de 6 a 10 & 49 & $25,00 \%$ \\
\hline de 11 a 15 & 49 & $25,00 \%$ \\
\hline de 16 a 20 & 59 & $30,10 \%$ \\
\hline de 21 a 25 & 16 & $8,16 \%$ \\
\hline de 25 a más & 4 & $2,04 \%$ \\
\hline Total & 196 & $100,00 \%$ \\
\hline
\end{tabular}

En la tabla 11, se presenta la información de la categoría de análisis referida a la cantidad de páginas incluida en cada número publicado. La mayor cantidad de números se concentra en el rango de 38 a 75 páginas, que corresponde a 9 números (32,14\% del total). En segundo lugar, se ubican 7 artículos (25\% del total) en el rango de 101 a 125 páginas. En tercer lugar, se ubican 6 números (21,43\% del total) en el rango de 76 a 100 páginas. Cabe mencionar que las publicaciones con menor número de páginas se concentran en las 10 primeras ediciones de la revista, mientras que, en las diez últimas ediciones, el contenido se ubica por encima de las 100 páginas. El promedio de páginas por número se ubica en 95 , número que se encuentra alineado a lo descrito en los resultados mostrados en la tabla 10 .

Otra categoría de análisis establecida corresponde a las referencias bibliográficas incluidas en cada artículo, información que se presenta en la tabla 12. En primer lugar, se encontraron 56 artículos $(28,57 \%$ del total) con 31 o más referencias bibliográficas. En segundo lugar, se ubican 29 artículos (14,80\% del total) en el rango de 6 a 10 referencias. En tercer lugar, se encuentran dos grupos con 23 artículos (11,73\% del total en cada caso) con los rangos de 1 a 5 y 26 a 30 referencias. Los artículos con menor número de referencias bibliográficas se ubican en las primeras ediciones; por otro lado, los que incluyen mayor cantidad se ubican, por lo general, en los últimos números. Otro resultado relevante se refiere al promedio de referencias bibliográficas por artículo, que corresponde a 24 referencias. En la mayoría de casos, se recurre a referencias internacionales y no locales, lo que concuerda con los resultados que se describen en el análisis de las investigaciones incluidas en la revisión de la literatura.

Tabla 11. Páginas por número

\begin{tabular}{|c|c|c|}
\hline Páginas por número & Números & $\%$ \\
\hline de 38 a 75 & 9 & $32,14 \%$ \\
\hline de 76 a 100 & 6 & $21,43 \%$ \\
\hline de 101 a 125 & 7 & $25,00 \%$ \\
\hline de 126 a 150 & 4 & $14,29 \%$ \\
\hline de 150 a más & 2 & $7,14 \%$ \\
\hline Total & 28 & $100,00 \%$ \\
\hline
\end{tabular}

Tabla 12. Referencias bibliográficas por artículo

\begin{tabular}{|c|c|c|}
\hline Referencias bibliográficas & Artículos & $\%$ \\
\hline Ninguna & 6 & $3,06 \%$ \\
\hline de 1 a 5 & 23 & $11,73 \%$ \\
\hline de 6 a 10 & 29 & $14,80 \%$ \\
\hline de 11 a 15 & 21 & $10,71 \%$ \\
\hline de 16 a 20 & 20 & $10,20 \%$ \\
\hline de 21 a 25 & 18 & $9,18 \%$ \\
\hline de 26 a 30 & 23 & $11,73 \%$ \\
\hline de 31 a más & 56 & $28,57 \%$ \\
\hline Total & 196 & $100,00 \%$ \\
\hline
\end{tabular}

Contabilidad y Negocios (15) 30, 2020 / ISSN 1992-1896 
La siguiente categoría de análisis se refiere a la cantidad de referencias bibliográficas incluidas por cada número publicado, resultados que se pueden observar en la tabla 13. Se aprecia que 8 números publicados tienen el mayor rango de referencias bibliográficas por número con 251 referencias o más $(28,57 \%$ del total). En segundo lugar, se encuentran 6 números con el rango entre 51 y 100 referencias $(21,43 \%$ del total). El tercer lugar es compartido por dos grupos de 5 números cada uno, en los rangos de 29 a 50 y 151 a 200 referencias $(17,86 \%$ del total en cada caso). Asimismo, el promedio de referencias bibliográficas por número se ubica en 172 .

Tabla 13. Referencias bibliográficas por número

\begin{tabular}{|c|c|c|}
\hline Referencias bibliográficas & Números & $\%$ \\
\hline de 29 a 50 & 5 & $17,86 \%$ \\
\hline de 51 a 100 & 6 & $21,43 \%$ \\
\hline de 101 a 150 & 2 & $7,14 \%$ \\
\hline de 151 a 200 & 5 & $17,86 \%$ \\
\hline de 201 a 250 & 2 & $7,14 \%$ \\
\hline de 251 a más & 8 & $28,57 \%$ \\
\hline Total & 28 & $100,00 \%$ \\
\hline
\end{tabular}

En la tabla 14 se presentan los resultados relacionados al tiempo de aprobación de los artículos publicados. Se puede observar que 48 artículos (24,49\% del total) se ubican en el rango de evaluación de 31 a 60 días. En segundo lugar, se encuentran 40 artículos (20,41\% del total) en el rango de aprobación de 61 a 120 días. En el tercer lugar, se ubican 38 artículos (19,39\% del total) en el rango de 181 a 365 días. Asimismo, se ha identificado que el promedio de días en que se realiza la aprobación de un artículo corresponde a 121 días. En las publicaciones analizadas en la revisión de la literatura, no se cuenta con información similar para realizar una comparación con esta categoría de análisis, pero los resultados evidencian el rigor en el tiempo dedicado a la revisión de los artículos evaluados para su publicación.

Tabla 14. Tiempo de aprobación

\begin{tabular}{|c|c|c|}
\hline Rango de días & Artículos & $\%$ \\
\hline de 1 a 15 & 25 & $12,76 \%$ \\
\hline de 16 a 30 & 12 & $6,12 \%$ \\
\hline de 31 a 60 & 48 & $24,49 \%$ \\
\hline de 61 a 120 & 40 & $20,41 \%$ \\
\hline de 121 a 180 & 24 & $12,24 \%$ \\
\hline de 181 a 365 & 38 & $19,39 \%$ \\
\hline de 365 a más & 9 & $4,59 \%$ \\
\hline Total & 196 & $100,00 \%$ \\
\hline
\end{tabular}

Fuente: Elaboración propia.

Con la finalidad de realizar una identificación de la autoría de los artículos a partir de género, se presenta la información de la tabla 15 . Por un lado, se observa que 253 autores $(66,75 \%$ del total) son de género masculino, lo que corresponde a la mayoría. Por otro lado, 126 autoras $(33,25 \%$ del total) son de género femenino. A medida que ha ido avanzando el tiempo, se muestra mayor presencia de autoras en las publicaciones de la revista. Estos resultados son similares a los obtenidos en las investigaciones analizadas en la revisión de la literatura, pues también se evidencia que el género femenino empieza a tener una mayor presencia con el paso de los años.

Tabla 15. Autores por género

\begin{tabular}{|c|c|c|}
\hline Género & Autores & $\%$ \\
\hline Femenino & 126 & $33,25 \%$ \\
\hline Masculino & 253 & $66,75 \%$ \\
\hline Total & 379 & $100,00 \%$ \\
\hline
\end{tabular}

Finalmente, en la tabla 16, se presentan los resultados de la categoría de análisis que identifica la filiación institucional. Se han considerado a las instituciones 
que figuran de 5 veces a más. En primer lugar, 152 autores $(40,11 \%$ del total) indican encontrarse afiliados a universidades que se ubican por debajo de las 5 menciones. En segundo lugar, 69 autores (18,21\% del total) mencionan a la PUCP como institución afiliada. En tercer lugar, se encuentra la Universidade Federal de Santa Catarina con 49 autores (12,93\% del total). Por último, se encuentra la Universidade Regional de Blumenau con 20 autores (5,28\% del total). Con respecto a esta categoría, también se encontraron resultados similares a los de las investigaciones analizadas en la revisión de la literatura, pues, en ambos casos, se observa mayor cantidad de autores que instituciones a las que pertenecen los mismos.

\section{Tabla 16. Universidad o institución de autores}

\begin{tabular}{|l|c|c|}
\hline \multicolumn{1}{|c|}{ Universidad } & Autores & $\%$ \\
\hline $\begin{array}{l}\text { Otras universidades o instituciones (menor } \\
\text { a cinco) }\end{array}$ & 152 & $40,11 \%$ \\
\hline PUCP & 69 & $18,21 \%$ \\
\hline Universidade Federal de Santa Catarina & 49 & $12,93 \%$ \\
\hline Universidade Regional de Blumenau & 20 & $5,28 \%$ \\
\hline Universidad de Valencia & 15 & $3,96 \%$ \\
\hline Universidad de Zaragoza & 14 & $3,69 \%$ \\
\hline Universidad de Vigo & 11 & $2,90 \%$ \\
\hline Universidad de Bío-Bío & 10 & $2,64 \%$ \\
\hline Universidade Federal do Rio de Janeiro & 8 & $2,11 \%$ \\
\hline Universidad de Barcelona & 7 & $1,85 \%$ \\
\hline Universidad ORT & 6 & $1,58 \%$ \\
\hline Universidad de Granada & 6 & $1,58 \%$ \\
\hline Universidad Autónoma de Madrid & 6 & $1,58 \%$ \\
\hline Universidad Autónoma de Bucaramanga & 6 & $1,58 \%$ \\
\hline Total & 379 & $100,00 \%$ \\
\hline
\end{tabular}

Tras presentar los resultados de las categorías de análisis, se observa una estrecha relación con el análisis y presentación de las investigaciones incluidas en la

revisión de la literatura de este artículo. En líneas generales, se han encontrado coincidencias con respecto a la evolución natural de una publicación científica, y la exigencia del proceso y selección de los artículos publicados. Contabilidad y Negocios, al igual que los casos estudiados en las investigaciones referidas, concentra el mayor número de publicaciones en el área de administración, contabilidad, y banca y finanzas. Se manifiesta una menor concentración de artículos sobre contabilidad de gestión, auditoría, tributación y áreas afines. Asimismo, se observa una similitud en la evolución de las metodologías aplicadas para la elaboración de los artículos; en la cantidad de autores por artículo publicado; y en la mayor presencia de autores de género masculino, aunque con una mejora progresiva en el posicionamiento del género femenino.

\section{Conclusiones y recomendaciones}

La contabilidad y la gestión de negocios están evolucionando y consolidándose en Perú y a nivel global de manera general. Siguiendo esa línea, la producción científica sobre estas áreas también está cambiando. En el caso de la revista Contabilidad y Negocios, se constataron cambios en el perfil de los autores, las bases teóricas utilizadas y los tipos de estudios realizados. El alcance de la revista se ha ampliado con autores y citas de países como Perú, Brasil, España, Colombia, Chile, México, Ecuador, Uruguay, Portugal, Argentina, Cuba, Estados Unidos, Venezuela, China, Costa Rica, Japón y Nicaragua. Los temas más relevantes en el período analizado en la revista fueron administración de empresas, actualidad contable y normas contables, banca y finanzas, contabilidad de gestión, auditoría, y tributación. Así, se pueden constatar posibles contribuciones teóricas y prácticas de las investigaciones en estos temas tanto en la formación de profesionales como con la gestión de 
negocios. En suma, la revista Contabilidad y Negocios se ha consolidado como una importante revista científica tanto en Perú como en Iberoamérica. Asimismo, en los últimos años, ha alcanzado mayor visibilidad, y ha manifestado incremento en la cantidad y calidad de las colaboraciones de sus autores. En tal sentido, el análisis de los resultados permite presentar las siguientes conclusiones:

a) Las principales áreas del conocimiento en que se concentra la producción científica, reflejada en 196 artículos publicados en los 28 números, muestran una alta inclinación hacia temas de administración, contabilidad financiera, finanzas y contabilidad de gestión.

b) La metodología más utilizada (en aproximadamente las tres cuartas partes de los artículos publicados en la revista) es de tipo descriptiva. Para que la revista logre su incorporación en bases de datos con un mayor índice de impacto, como Journal Citation Reports (JCR) o Scimago Journal Rank (SJR), necesita aceptar y publicar artículos con un mayor rigor en el método científico aplicado.

c) Los artículos incorporados en los 28 números publicados evidencian que la revista tiene una política editorial abierta. Asimismo, no demuestra un comportamiento endogámico, ya que la mayor parte de artículos proceden de otras universidades y de países distintos al de la afiliación institucional de la revista.

d) La revista Contabilidad y Negocios debe aprovechar la ventaja que se genera por el interés de investigadores y docentes de otros países en publicar sus investigaciones en la revista, lo que permite incrementar la visibilidad de las publicaciones y la posibilidad de incrementar el nivel de citas de los artículos publicados. e) Con el fin de lograr una paridad de género en las publicaciones, es recomendable que el Comité Editorial establezca políticas e implemente estrategias para incrementar el número de autoras de género femenino.

f) La revista cuenta con algunos indicadores que evidencian una mejora importante en la cantidad y calidad de los artículos, como, por ejemplo, el mayor número de páginas por artículo, el incremento en el número de referencias bibliográficas por artículo y la cantidad de autores por artículo, entre otros.

Este artículo está limitado al análisis bibliométrico de los artículos de la propia revista Contabilidad y Negocios. Por lo tanto, busca comprender en un medio de comunicación específico su contribución para la academia, por lo que no extrapola para otros medios. Además, se ha limitado a los aspectos bibliométricos de los artículos y sus temas. De esta manera, el análisis propuesto en este artículo revela la importancia de monitorear la producción técnico-metodológica de la revista Contabilidad y Negocios por parte de la comunidad académica, ya que enriquece el proceso de internacionalización deseado por la revista, y la inserción de investigadores y profesionales de contabilidad y administración en publicaciones. Por ello, para trabajos futuros se recomienda realizar comparaciones con otras revistas y bases de investigación; es decir, se sugiere confrontar la revista con la experiencia internacional a partir del análisis de la cooperación entre los diferentes países y el análisis de citas de las obras.

\section{Referencias}

Bordons, M. \& Zulueta, A. (1999). Evaluación de la actividad científica a través de indicadores bibliométricos. Revista Española de Cardiología, 52(10), 790-800. https://doi.org/10.1016/S0300-8932(99)75008-6 
Brunozi, A.C.; Emmerdoerfer, M.L.; Abrantes, L.A.; Klein, T.C. (2011). Revista Contabilidade \& Finanças USP: uma análise do perfil da produção científica de 1989 A 2009. Revista Universo Contábil, 7(4), 39-59.

Carvalho, A.J., Pierri, M.A., Lunkes, R.J. \& Gasparetto, V. (2016). Management Accounting: A Bibliographic Study in Top International Accounting Journal's. Sociedade, Contabilidade e Gestão, 11(2), 155-174. https://doi.org/10.21446/scg_ufrj.v11i2.13384

Contabilidad y Negocios. (2020). Lima: Departamento de Ciencias Contables. Pontificia Universidad Católica del Perú. Recuperado de http://revistas.pucp.edu.pe/ index.php/contabilidadynegocios

Duque, P. \& Cervantes-Cervantes, L.-S. (2019). Responsabilidad social universitaria: una revisión sistemática y análisis bibliométrico. Estudios Gerenciales, 35(153), 451-464. https://doi.org/10.18046/j. estger.2019.153.3389

Giménez, E. (2018). La evaluación de las Humanidades y de las Ciencias Sociales en revisión. Revista Española de Documentación Cientifica, 41(3), 1-15. https://doi. org/10.3989/redc.2018.3.1552

Imasato, T., Perlin, M. S. \& Borenstein, D. (2017). Análise do Perfil dos Acadêmicos e de suas Publicaçóes Científicas em Administração. Revista de Administraçâo Contemporânea, 21(1), 62-83. https://doi. org/10.1590/1982-7849rac2017150329

Jiménez, M. A., Gasparetto, V., Lunkes, R. J. \& Schnorrenberger, D. (2017, julio-diciembre). Contabilidad de gestión en universidades públicas de Colombia: un análisis de los planes de estudio y contenidos académicos. Revista Iberoamericana de Contabilidad de Gestión, XV(30), 1-14.

Kremer, A., Luz, M. B. L., Suave, R. \& Lunkes, R. J. (2014). Pesquisa científica em contabilidade gerencial: um estudo em periódicos de negócios. Revista de Contabilidade da UFBA, 8(1), 54-69.
Krivogorsky, V. \& Ballam, M. J. (2019). Teaching an International Course in the Business School: A New Blended Approach. Innovations in Education and Teaching International, 56(3), 330-340. https://doi.org/1 $0.1080 / 14703297.2018 .1498367$

Lunkes, R., Ripoll Feliu, V. M. \& Silva da Rosa, F. S. (2013). Study of Published Articles on Management Accounting in Brazil and Spain. Revista Contabilidade \& Finanças, 24(61), 11-26. https://doi.org/10.1590/ S1519-70772013000100003

Pereira, A. C. D., Souza, P. \& Lunkes, R. J. (2013). Análise bibliométrica das publicaçóes de contabilidade em revistas de turismo de 1992 a 2012. Revista Acadêmica Observatório de Inovação do Turismo, VIII(1), 39-61. https://doi.org/10.12660/oit.v8n1.32089

Pérez, M. \& Lutsak-Yaroslava, N. (2017). La producción científica sobre la innovación social para el desarrollo local. Una revisión bibliométrica. Revista Prisma Social, (19), 146-182.

Reina, D. R. M., Ensslin, S.R., Ensslin, L. \& Reina, D. (2016). Seleção e análise do perfil da produção científica sobre o tema seleção de projetos. Revista REGE, 21(1), 3-25. https://doi.org/10.5700/rege516

Ribeiro, H. C. M. (2013). Revista Contemporânea de Contabilidade: uma análise do perfil da produção acadêmica durante o período de 2004 a 2012. Revista Contemporânea de Contabilidade, 10(20), 03-28. https://doi.org/10.5007/2175-8069.2013v10n20p3

Ribeiro, H. C. M. \& Costa, B. K. (2013). Brazilian Administration Review: uma análise do perfil da produção acadêmica científica no período de 2004 a 2012 sob a ótica da rede social e da bibliometria. Revista de Ciências da Administraçâo, 15(37), 65-81. https://doi. org/10.5007/2175-8077.2013v15n37p65

Rosa, R. A. \& Romani-Dias, M. (2019). A Presença e o Impacto de Periódicos Brasileiros da Área de Administração, Contabilidade e Turismo em Bases Científicas. 
Revista Eletrônica de Ciência Administrativa, 18(3), 327348. https://doi.org/10.21529/RECADM.2019014

Sánchez-Perdomo, R., Rosario-Sierra, M., Herrera-Vallejera, D., Rodríguez-Sánchez, Y. \& Carrillo-Calvet, H. (2018). Revisión bibliométrica de las Ciencias de la Información en América Latina y el Caribe. Investigación Bibliotecológica, (Número Especial de Bibliometría), 79-100. https://doi.org/10.22201/ iibi.24488321xe.2017.nesp1.57886

Smolski, F. M. S., Dalcin, D., Visentini, M. S. \& Bamberg, J. (2017). Análise do perfil da produção científica da Revista de Administração Pública (RAP) no período
2003-16. Revista De AdministraçâoPública, 51(6),11391163. https://doi.org/10.1590/0034-761220170046

Spraakman, G. \& Jackling, B. (2014). A Conceptual Framework for Learning Management Accounting. Accounting Perspectives, 13(1), 61-81. https://doi. org/10.1111/1911-3838.12024

Fecha de recepción: 25 de junio de 2020 Fecha de aceptación: 13 de octubre de 2020 Correspondencia: odiaz@pucp.edu.pe fabriciasrosa@hotmail.com montes.mg@pucp.edu.pe 